\title{
Vers le milieu de recherche pour soutenir les élèves : une CAP sur le vocabulaire
}

\section{HRONIQUE • Milieu scolaire}

\section{Introduction}

De plus en plus d'élèves allophones sont accueillis dans les classes au Québec et le développement du vocabulaire en français représente un défi important pour le personnel enseignant (Fleury, 2013). C'est le constat qu'ont fait les membres d'une communauté enseignante dans une école primaire dont la population est majoritairement allophone pour ensuite se mobiliser avec comme objectif de trouver des moyens éprouvés par la recherche visant l'enrichissement du vocabulaire chez leurs élèves. En effet, convaincues des bénéfices engendrés par un vocabulaire plus étendu au regard de la compréhension en lecture ou des travaux d'écriture des élèves, la direction d'école et les enseignantes du $2^{\mathrm{e}}$ cycle ont formé une communauté d'apprentissage professionnelle (CAP). Après une consultation intensive des pratiques avérées efficaces, la CAP s'est unanimement arrêtée sur une pratique : le développement de la conscience morphologique en travaillant les racines, les préfixes et les suffixes avec les enfants. Le présent article rend compte de l'expérience pédagogique vécue à cette école, des progrès constatés chez les élèves ainsi que des conditions gagnantes qui ont rapproché les milieux scolaires et scientifiques au cours de l'implantation d'un programme d'enseignement issu de la recherche visant le développement de la conscience morphologique.

\section{Contexte ou histoire de la CAP}

\section{Besoins du milieu}

C'est à la fin de l'année scolaire 2017-2018 que le personnel enseignant et la direction se sont réunis pour se pencher sur les besoins des élèves de l'école. L'objectif était de mieux répondre aux besoins afin de parvenir à réduire le nombre d'élèves identifiés comme éprouvant des difficultés d'apprentissage. Ensemble, ils ont dégagé que le bagage de vocabulaire en français demeurait assez pauvre chez les enfants de la maternelle jusqu'à la fin du primaire probablement parce que la majorité des élèves 
revenait à leur langue maternelle aussitôt de retour dans leur milieu respectif. Or, puisqu'il suffit de ne pas connaitre seulement $2 \%$ des mots d'un texte pour que sa compréhension devienne ardue (Fezjo, Godard et Laplante, 2014) le besoin commun ciblé fut l'amélioration du vocabulaire des élèves de l'école dont une forte majorité évolue au sein d'une famille allophone.

\section{Choix de la compétence à développer}

L'orthopédagogue de l'école s'est alors penchée sur la recherche afin de trouver les moyens les plus efficaces d'intervenir pour enrichir le vocabulaire chez les élèves. C'est le développement de la conscience morphologique qui a retenu son attention puisque les études récentes démontrent que cette pratique pédagogique favorise les habiletés de lecture et d'écriture chez les enfants, et ce, plus particulièrement chez les élèves éprouvant des difficultés d'apprentissage (Bowers, Kirby et Deacon, 2010). En fouillant davantage sur le sujet, il s'est avéré intéressant d'apprendre qu'à partir du $2^{\mathrm{e}}$ cycle du primaire $60 \%$ des mots composant les textes scolaires peuvent être compris grâce à leur structure morphologique (Nagy et Anderson, 1984, cités dans Fejzo, 2016). Aussi, il appert que la connaissance des préfixes et des suffixes d'un mot permet de comprendre trois ou quatre nouveaux mots appartenant à la même famille (Fejzo, 2016) contribuant ainsi à l'amélioration du vocabulaire chez les élèves. Finalement, un aspect non négligeable lié au développement de la conscience morphologique que Fejzo a constaté dans le cadre de ses recherches est qu'il amène aussi les élèves à se questionner et analyser les mots inconnus rencontrés dans un texte avant même de songer à consulter un dictionnaire.

\section{Sélection du programme en conscience morphologique}

C'est en s'appuyant sur ces faits que le programme d'enseignement issu de la recherche Histoire de famille (Fejzo, 2016) s'adressant principalement aux élèves du $2^{\mathrm{e}}$ cycle du primaire a été ciblé comme outil de choix à présenter aux enseignantes. Il s'agit d'un programme développé pour un enseignement universel en classe, donc au palier 1 du modèle de réponse à l'intervention (RàI) et dont les résultats ont été prouvés efficaces dans le cadre de recherches et d'expérimentations. C'est aussi à ce moment qu'une CAP regroupant cinq enseignantes du $2^{\mathrm{e}}$ cycle, une orthopédagogue ainsi que la direction a été formée.

Or, l'orthopédagogue étant déjà très prise par un horaire chargé, la direction a consenti à embaucher une nouvelle ressource à raison d'une demi-journée par semaine pour prendre le relai à la coordination de ce projet. Une orthopédagogue supplémentaire s'est donc jointe à l'équipe de la CAP. Dans un premier temps, elle s'est chargée d'organiser une présentation soutenant le choix de la compétence à développer ainsi que celui de l'outil d'enseignement prouvé efficace par la recherche. À l'issue de cette présentation, directrice et enseignantes ont été invitées à se mettre dans la peau des élèves alors que l'orthopédagogue prenait le rôle d'enseignante pour faire vivre la première activité du programme en entier aux membres de la CAP. Par la suite, les enseignantes ont été invitées à se prononcer sur l'outil proposé et sur leur intérêt à l'ajouter à leurs pratiques pédagogiques. D'emblée, toutes ont accepté sachant que la CAP leur assurerait soutien et temps de travail en équipe nécessaires au développement du projet. 


\section{Mise en place}

Dès lors, l'organisation des périodes d'enseignement et de la suite du projet s'est mise en branle, le tout orchestré par l'orthopédagogue coordonnatrice, mais toujours en concertation avec tous les membres de la CAP. Le calendrier des rencontres de l'équipe de la CAP $2^{\mathrm{e}}$ cycle a été établi et celui-ci a non seulement permis de fixer des objectifs dans le temps, mais aussi de prévoir les besoins en matière de suppléances. Un groupe d'échange électronique a été créé et un journal de bord accessible à tous a été produit pour favoriser la consignation des bons coups, des questions ou des commentaires tout au long du projet. Un travail de coopération entrepris par les enseignantes elles-mêmes leur a permis de partager les tâches de préparation matérielle des dix premières activités du programme afin de s'assurer que tous les groupes disposaient du même matériel. Les élèves ont par la suite été soumis à un prétest mesurant la conscience morphologique et le vocabulaire et les résultats ont été compilés avec comme objectif de les comparer à un post-test à administrer à la suite de l'enseignement du programme. C'est au moment de choisir un prétest adéquat que l'orthopédagogue coordonnatrice du projet est entrée en communication avec l'auteure du programme Histoire de famille pour s'assurer de la pertinence de l'outil d'évaluation à utiliser au regard des progrès à évaluer une fois l'enseignement terminé. Dès lors, madame Fejzo également chercheuse à l'UQAM, s'est vivement intéressée au projet de l'école et a collaboré avec la coordonnatrice tout au long de son déroulement.

L'enseignement du programme Histoire de famille a commencé en janvier alors que l'orthopédagogue coordonnatrice a animé la première activité en collaboration avec les enseignantes des cinq classes sur une journée. Ces dernières ont poursuivi l'enseignement des activités subséquentes selon un horaire qui leur convenait considérant l'échéance planifiée pour la fin avril. Deux rencontres de suivi de la CAP ont eu lieu durant cette période et ont été favorables pour échanger, se questionner et réajuster le calendrier d'implantation. Une présentation du projet à l'ensemble du personnel de l'école a été faite en février et celle-ci comprenait des suggestions d'autres programmes visant l'enrichissement du vocabulaire issus de la recherche afin de susciter l'intérêt des enseignantes d'autres cycles à se réunir en CAP pour les explorer. Finalement, un post-test a été administré et les enseignantes ont été formées pour en faire la correction et entrer les données dans les grilles conçues par la coordonnatrice.

\section{Résultats}

En fin d'année, les résultats ont été présentés et expliqués dans le cadre d'une rencontre de la CAP. Ceux-ci ont démontré que tous les élèves s'étaient nettement améliorés pour les deux volets ciblés par l'enseignement : la conscience morphologique et le vocabulaire. L'amélioration la plus marquée a été observée au niveau du vocabulaire chez les élèves de $3^{\mathrm{e}}$ année. De plus, les résultats ont permis de constater des progrès au niveau de la conscience syntaxique. D'autre part, une analyse comparative avec des groupes de recherche ayant participé à une étude portant sur la conscience morphologique (Fejzo, 2014-2017) a permis de dégager que les gains en vocabulaire ont été plus importants chez les élèves de nos groupes. Ce constat permet d'émettre l'hypothèse que les effets sont plus grands lorsque les enseignantes elles-mêmes intègrent les activités pédagogiques dans leur pratique plutôt que lorsque ce sont des assistantes ou assistants de recherche qui interagissent ponctuellement avec les élèves dans le cadre d'une recherche. 


\section{Conditions qui ont mené au rapprochement des deux milieux}

Réfléchissant aux conditions qui ont mené au rapprochement du milieu scolaire et de la recherche universitaire dans le cadre de notre projet, nous avons dégagé les conditions suivantes :

\section{Des praticiens réflexifs et orientés vers la recherche}

Le rapprochement est amorcé par les praticiens réflexifs, soit des orthopédagogues et des enseignantes qui se tiennent au courant des avancées de la recherche et qui se basent sur celles-ci dans leurs choix pédagogiques.

\section{Un leadeurship pédagogique de la direction}

La direction d'école reconnaissant l'importance de la collaboration et de la coopération a favorisé la création d'une CAP en prévoyant un budget spécifique afin d'offrir du temps de travail et de réflexion en équipe aux enseignantes en dehors de leur classe.

\section{Une ressource humaine pour soutenir les enseignants}

Une condition gagnante est lorsqu'une direction d'école reconnait que l'implantation d'une nouvelle pratique et la mesure de son succès parallèlement à l'enseignement prévu demandent soutien et accompagnement d'une ressource supplémentaire. Dans le cadre de ce projet, la direction a dégagé le budget nécessaire à l'embauche d'une orthopédagogue contractuelle à raison d'une demi-journée par semaine pour toute l'année : tout le volet organisationnel de l'implantation du programme ainsi que le volet d'évaluation des progrès d'élèves et la formation des enseignantes à ce sujet ont été pris en charge par cette ressource toujours après des décisions ayant fait consensus lors des périodes de travail de la CAP.

\section{Un contact avec le milieu de recherche}

Le rapprochement est facilité quand un milieu scolaire considère le milieu de recherche comme un allié potentiel, important et accessible pour la bonne marche de son projet d'expérimentation et d'implantation et également quand, en parallèle, une équipe de recherche fait preuve d'ouverture et de disponibilité pour collaborer et soutenir le milieu scolaire dans ses démarches. Nul doute que cette volonté commune de collaboration des deux milieux a permis le succès du projet faisant l'objet de cet article.

\section{L'intégration dans un projet de recherche}

Un intérêt commun né d'une expérience vécue au sein d'un projet peut mener à de nouvelles collaborations entre les milieux scolaire et universitaire. Comme le dit Daviau (2018), il est important que les questionnements des enseignants soient à l'origine des recherches menées par les universitaires en collaboration avec le milieu. C'est ce qui a favorisé la conception du projet L'élaboration d'un dispositif axé sur la conscience morphologique par les enseignants en collaboration avec les chercheurs et ses 
effets sur le vocabulaire et la compréhension en lecture chez des élèves allophones des $2^{e}$ et $3^{e}$ cycles du primaire (Fejzo, Laplante, Gonnerman et Côté, FRQSC Actions concertées 2019-2023). L'entière collaboration des enseignantes de l'école dans cette nouvelle aventure pédagogique est l'indicateur d'une formule gagnante.

\section{Note}

1 L'auteure tient à remercier ses collègues membres de la CAP qui ont participé à la phase de révision de cet article: Julie Grothé, orthopédagogue et directrice adjointe (CSMV) ainsi que Maryse Beaupré, Nathalie Cloutier et Fanny Dion, enseignantes au $2^{e}$ cycle du primaire (CSMV).

\section{Conclusion}

Né de l'initiative d'une équipe-école regroupée en CAP, le projet de développement du vocabulaire chez les élèves allophones de l'école a été bonifié par la collaboration volontaire d'une chercheuse très intéressée par le sujet. Ainsi, le milieu de la recherche a pu constater les effets encore plus positifs engendrés par une collaboration plus étroite et l'implication réelle des enseignants dans l'expérimentation de nouvelles pratiques. Par ailleurs, la recherche à venir viendra documenter une expérience pédagogique entièrement conçue en ce sens. Mais à la lumière de l'expérience vécue dans une école de la CSMV, nous pouvons émettre l'hypothèse qu'une collaboration accrue entre le milieu scolaire et celui de la recherche représente une condition gagnante autant pour les enseignantes et enseignants que pour les élèves.

Est-ce à dire que, dans le futur, les changements de pratiques pourraient être envisagés non seulement par le milieu scolaire en vertu de résultats d'expérimentation probants, mais aussi par les chercheurs universitaires qui pourraient en venir à impliquer davantage les enseignants dans le développement et l'utilisation d'outils de recherche?

\section{Références}

Bowers, P. N., Kirby, J. R. et Deacon, S. H. (2010). The effects of morphological instruction on literacy skills: A systematic review of the literature. Review of Educational Research, (80)2, 144-179. http://dx.doi.org/10.3102/0034654309359353

Daviau, C. (2018). Avis de recherche. Formation et profession, 26(3), 109-111. http://dx.doi.org/10.18162/fp.2018.a156

Fejzo, A. (2016). Histoire de famille (Trousse pédagogique). Bromont, QC : Fino Éducation.

Fejzo, A., Godard, L. et Laplante, L. (2014). L'analyse morphologique : pierre angulaire dans l'enrichissement du vocabulaire. Québec français, (171), 80-82. Repéré à CHRO MILIEU.rtf https://www.erudit.org/fr/revues/qf/2014-n171-qf01221/71231ac/

Fleury, R. (2013). Favoriser l'usage du français en milieu plurilingue : défis et réussites. Québec français, (168), 48-49. Repéré à https://www.erudit.org/fr/revues/qf/2013-n168-qf0476/68661ac/

\section{Pour citer cet article}

Latour, D. (2020). Vers le milieu de recherche pour soutenir les élèves : une CAP sur le vocabulaire. Formation et profession. 28(1), 142-146. http://dx.doi.org/10.18162/fp.2020.a196 